\title{
Underwater Acoustic Communications in Time-Varying Dispersive Channels
}

\author{
Iwona Kochańska \\ Gdańsk University of Technology \\ ul. Narutowicza 11/12, \\ 82-233 Gdańsk, Poland \\ Email: \\ iwona.kochanska@eti.pg.gda.pl
}

\author{
Jan Schmidt \\ Gdańsk University of Technology \\ ul. Narutowicza 11/12, \\ 82-233 Gdańsk, Poland \\ Email: \\ jan.schmidt@eti.pg.gda.pl
}

\author{
Mariusz Rudnicki \\ Gdańsk University of Technology \\ ul. Narutowicza 11/12, \\ 82-233 Gdańsk, Poland \\ Email: \\ mariusz.rudnicki@eti.pg.gda.pl
}

\begin{abstract}
Underwater acoustic communication (UAC) system designers tend to transmit as much information as possible, per unit of time, at as low as possible error rate. However, the bit rate achieved in UAC systems is much lower than for wire or radio-communication systems. This is due to disadvantageous properties of the UAC channels, namely the sea and inland waters. Estimation of UAC channel transmission properties is possible within a limited bandwidth and temporal resolution. Thus, the UAC physical layer of data transmission is designed on the basis of roughly estimated channel parameters, or assuming the worst possible conditions. The paper presents the methodology of adapting UAC signaling schemes to tough underwater propagation conditions, through an example of two communication systems designed and developed at the Gdansk University of Technology.
\end{abstract}

\section{INTRODUCTION}

$\mathrm{T}$ HE Department of Marine Electronic Systems, the Faculty of Electronics, Telecommunications and Informatics Gdańsk University of Technology has a long and rich tradition in the processing of acoustic signals used in underwater systems. The main areas of interest are hydrolocation systems, namely sonar systems [1-6], and UAC systems [79]. The latter are particularly vulnerable to interference, due to tough propagation conditions, causing a time dispersion and time variability in the acoustic signals transmitting the information.

Due to a wide range in the transmission properties of UAC channels, there are, in the world, only few standards used to define very slow communication. In deep-water channels, transmission rates of up to $100 \mathrm{kbps}$ can be achieved, while the same research centers offer much slower standards in shallow water channels, where reliable communication at a speed of $40-80 \mathrm{bps}$ is a significant achievement. Such differences in UAC systems performances is due, inter alia, to the large temporal uncertainty of underwater channel transmission characteristics [10].

Furthermore, depending on the communications system in question, there are different requirements for speed and the dependability of transmissions in acoustic links. It may be a case of: a) slow but reliable transmission with autonomous underwater vehicle's (AUV) control signals, b) slightly faster but still generally reliable measurement of data trans- mission from an underwater monitoring system or, c) maximum speed video transmissions from underwater cameras. Therefore, there is no one, single, typical, UAC problem. The physical layer of the data transmission should be adapted to the specific propagation conditions of the particular channel.

\section{TRANSMisSION PROPERTIES OF THE UAC CHANNEL}

\section{A. Propagation conditions}

The range of the UAC system is determined mainly by the absolute value of absorption attenuation, and varies in proportion to the square of the frequency of the system (except for the band between 0.5 to $5 \mathrm{kHz}$ and 200 to $1000 \mathrm{kHz}$, in which it grows more slowly). Excessive differences of attenuation, due to growth in range has a limiting effect on the bandwidth of the system and reduces its throughput.

Phenomenon which strongly impacts transmission properties of the UAC channel consists of reflections from the seabottom and the water's surface, as well as other objects present in water. This causes multipath propagation, and, in consequence, reception of both direct and delayed signals. This phenomenon also goes hand-in-hand with strong refraction, which is caused by a significant change in sound velocity as a function of depth. Both multipath propagation as well as refraction produce time dispersion in the transmitted signal, that can be measured as a parameter, known as multipath delay spread $\tau_{M}$. Moreover, due to multipath propagation phenomenon, selective fading of transmitted signal spectrum is observed. A maximum bandwidth not affected with selective fading is expressed as coherence bandwidth $B_{C}$

The movement of the UAC system's transmitter and receiver causes the Doppler Effect, resulting in the time-domain scaling of a natural broadband communication signal. This phenomenon also has a significant impact on the communication system's performance. Because of the relatively low velocity of propagation of acoustic waves in water, which is approximately $1500 \mathrm{~m} / \mathrm{s}$, the relative Doppler shift is approx. 200,000 times higher than in case of radio-communication systems. The impact of the Doppler Effect on the received signal in UAC channels is expressed as a scal- 
ing factor or - for narrowband signals - a maximum Doppler spread $v_{M}$.

UAC channel propagation conditions can change overtime. Depending on the phenomena under consideration, the variability of transmission properties can be of a range of several months (i.e. seasons), several days and hours (i.e. tides, times of day), minutes (i.e. internal waves), a few seconds (i.e. surface waves) as well as the order of milliseconds (that is to say: reflections, scattering) [7]. In designing the UAC physical layer it is essential to determine the time and frequency ranges over which the channel can be considered as stationary. This is defined by coherence time $T_{C}$ parameter [11].

\section{B. UAC channel transmission properties measurement}

The time-varying impulse response (TV-IR) $h(t, \tau)$ of the UAC channel is modeled as tapped delay line (Fig. 1). It is defined in the domain of two time variables: observation time $t$ and delay $\tau$. The values of $t$ indicate the moments of subsequent IR measurements while $\tau$ denotes the position on the time axis of successive samples of the IR in a single observation.

TV-IR is the basis for computation of transmission characteristics. If TV-IR fulfills the wide sense stationary uncorrelated scattering (WSSUS) assumption, it can describe the channel statistically, providing information on transmission parameters: multipath delay spread $\tau_{M}$, Doppler spread $v_{M}$, coherence time $T_{C}$ and coherence bandwidth $B_{C}$ [11]. The accuracy of instantaneous transmission parameters depends on the accuracy of channel impulse response estimation [12].

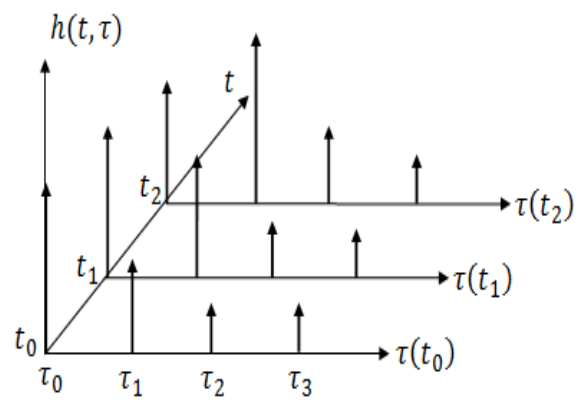

Fig 1. Tapped-delay line model of time-varying impulse response.

The measurement of impulse response is performed using the correlation method. As the probe signals, pseudorandom binary sequences (PRBSs) and linear frequency-modulated (LFM) chirps are used. A PRBS is the repetition of the maximal-length binary sequence $c_{m} \in\{-1,1\}$, modulated onto a binary phase-shift keyed waveform. Maximal-length binary sequences (known also as m-sequences) are widely used for communication synchronization, and transmissions of signals below the noise level [13-14]. A single "ping” of the passband PRBS of the center frequency $f_{C}$, bandwidth $B$, time duration $T$, m-sequence length $M$, and bit pulse shape $u(t)$ is given by:

$$
p(t)=\sin \left(2 \pi f_{c} t\right) \sum_{m=0}^{M-1} c_{m} u\left(t-\frac{m}{M} T\right),
$$

where $0 \leq t \leq T$ and $0 \leq m \leq M$, while a single "ping" of an LFM chirp signal is given by:

$$
p(t)=\sin \left(2 \pi\left[\left(f_{c}-\frac{B}{2}\right) t+\frac{B}{2 T} t^{2}\right]\right)
$$

The channel probe signal is constructed as a concatenation of $N$ pings:

$$
s(t)=\sum_{n=0}^{N-1} p(t-n T)
$$

As a result of correlative measurement, time-varying impulse response $h(t, \tau)$ is obtained. The duration $T$ and resolution $T / M$ of a single "ping" determines range and resolution of variable $\tau$. Moreover, the resolution of $t$ is equal to $1 / T$, while its range depends on number of "pings" and is equal to $N T$.

The uncertainty in time (and frequency) of probe signals is characterized by their ambiguity functions $A(\tau, v)$. It shows correlation filter response for a single ping as being a function of the time delay $\tau$ from the beginning of signal and frequency (Doppler) shift $v$ quantified for the center frequency:

$$
A(\tau, v)=\int_{-\infty}^{\infty} p(t) p^{*}(t-\tau) e^{2 \pi i v t} d t,
$$

where $0 \leq \tau \leq T$ and $-1 /(2 T)<v<1 /(2 T)$. Fig. 2. shows ambiguity functions of PRBS and LFM signals of duration of $T=256 \mathrm{~ms}$. At zero frequency shift, the ambiguity functions are the same as for PRBS and LFM, but in other areas they are very different. The PRBS offers high resolution in both delay and Doppler, but experiences clutter at frequency shift $v \neq 0$ [15].

a)

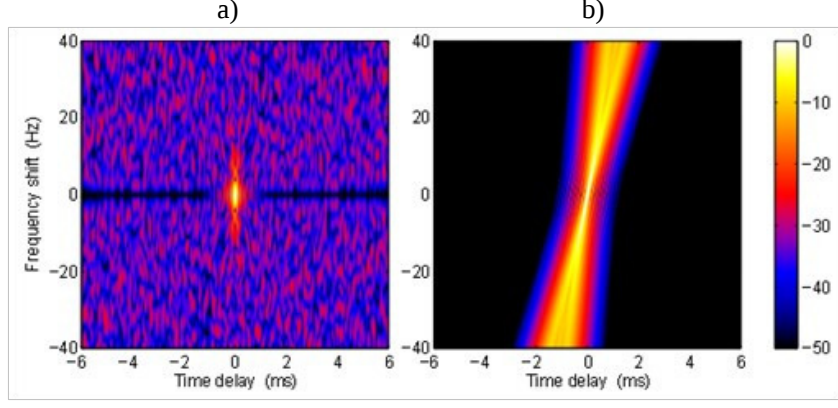

Fig 2. Ambiguity functions of PRBS (a) and LFM chirp signal (b) [15].

The chirp has a much stronger response than the PRBS for Doppler-shifted signals, but this Doppler insensitivity comes with a delay shift of $\Delta \tau=v \times T / B$. The LFM also shows some broadening in large frequency shifts [15].

\section{The UAC impulse response}

As a result of correlation measurement an estimate of TV-IR $h(t, \tau)$ is obtained. A single TV-IR estimate can be acquired but amounting to no more than the duration $T$ of the probe sequence, which should be adapted to the geome- 
try and the time variability of the channel. The channel should be sampled as often as possible to obtain a TV-IR estimate with a satisfactory time resolution. However, $T$ is limited by the coherence time $T_{C}$ of the channel. This limitation stems from the assumption that channel characteristics should remain unchanged for the duration of a single measurement in order to obtain a single estimate of the impulse response. At the same time, $T$ should be greater than the time dispersion of the channel expressed as multipath delay spread $\tau_{M}$.

Depending on the reciprocal of product of the coherence time $T_{C}$ and the coherence bandwidth $B_{C}$, known as the spreading factor $S F=1 / B_{C} T_{C}$, the UAC channel can be classified as being "underspread" or "overspread".

If , the $S F<1$ channel is assumed to be underspread. Such a channel can have a long impulse response which varies gradually, and this can be measured through the use of sufficiently long m-sequence or LMF signals. Underspread channels are also channels that change rapidly, but who possess a short TV-IR. A series of measurements through the use of short testing signals allows us to obtain knowledge on the nature of the variation in such channels.

If $S F>1$, the channel is called overspread. In this case, the channel has a long and rapidly varying impulse response. Measurement of the TV-IR of an overspread channel is extremely difficult and gives unreliable results, if not totally impossible [12]. Measurement with the use of a short sequence allows to examine variability in the channel, but the IR will be that of visible time-aliasing [15]. On the other hand, measurement with a long testing signal will result in the collection of true information on the distribution of subsequent multipath components along the time line. However, information on channel variability will be lost.

\section{WSSUS assumption}

Time-varying impulse response $h(t, \tau)$ is a starting point for the construction the UAC channel model. Due to the complexity of acoustic waves' propagation mechanism, building a deterministic description is a complex task. Therefore, a statistical approach is applied, assuming (as in the radiocommunications [11]) that the channel can be described with Rayleigh distribution, making the set of the time-varying channel impulse response $h(t, \tau)$ a two-dimensional Gaussian processes with a mean of zero. With this supposition, the characteristics of the channel will be a specification of second-order statistics and the autocorrelation function of the impulse response, in addition to the IR itself, will be a subject for further analysis.

The autocorrelation of two-dimensional impulse response $h(t, \tau)$ is a four-dimensional function:

$$
R_{h}(t, t+\Delta t, \tau, \tau+\Delta \tau)=E\left\{h^{*}(t, \tau) h(t+\Delta t, \tau+\Delta \tau)\right\}
$$

where $-T<\Delta \tau<T$ and $-N T<\Delta t<N T$. A channel is called wide-sense stationary (WSS) if the mean value of $h(t, \tau)$ is constant and $R_{h}(t, t+\Delta t, \tau, \tau+\Delta \tau)$ is stationary process in $t$ domain. Thus it can be reduce to a threedimensional function:

$$
R_{h}(t, t+\Delta t, \tau, \tau+\Delta \tau) \stackrel{\text { WSS }}{=} R_{h}(\Delta t, \tau, \tau+\Delta \tau)
$$

Additionally the uncorrelated scattering (US) assumption is fulfilled, when there is no correlation between the fading coming from different signal scatters. Thus the impulse response $h(t, \tau)$ is uncorrelated in $\tau$ domain and the autocorrelation function can be denoted as:

$$
R_{h}(t, t+\Delta t, \tau, \tau+\Delta \tau) \stackrel{U S}{=} R_{h}(t, t+\Delta t, \Delta \tau)
$$

The transfer function $H(t, f)$ of US impulse response has an autocorrelation function that is "stationary" in frequency domain $f$.

Under WSSUS assumption the autocorrelation function can be reduced to a two-dimensional function [16]:

$$
R_{h}(t, t+\Delta t, \tau, \tau+\Delta \tau) \stackrel{\text { wSSUS }}{=} R_{h}(\Delta t, \Delta \tau)
$$

The second order statistics are thus presumed to be time-invariant, and signal reflections reaching the receiver are treated as mutually uncorrelated.

In practical systems TV-IR is measured with with bandpass modulated signal, and than down-sampled into baseband. Thus, the resulting $h(t, \tau)=h_{I}(t, \tau)+h_{Q}(t, \tau)$ is complex time process, represented with real (in-phase) $h_{I}(t, \tau)$ and imaginary (quadrature) $h_{Q}(t, \tau)$ components. According to [17], for a WSS bandpass process, the in-phase and quadrature components are balanced in the sense that they have the same autocorrelation function. Also, the cross correlation of the in-phase and quadrature components must be an odd function for any pair of WSS processes. This property can be used to test whether $h(t, \tau)$ is WSS [18]. Testing US condition requires the calculation of the complex transfer function $H(t, f)$ as the Fourier transform of $h(t, \tau)$. Analysis of in-phase and quadrature components of $H(t, f)$, analogous to WSS test, is than performed.

\section{E. Transmission Characteristics}

Under the WSSUS assumption the scattering function $S(\nu, \tau)$ can be calculated as the Fourier transform in $t$ domain. It characterizes the mean amplitude of signal reflections reaching the receiver with the delay $\tau$ and Doppler Shift $v$ :

$$
S(v, \tau)=\int_{-\infty}^{\infty} h(t, \tau) e^{-j 2 \pi \Delta v \Delta t} d \Delta t
$$

where $-1 / 2 T<v<1 / 2 T$.

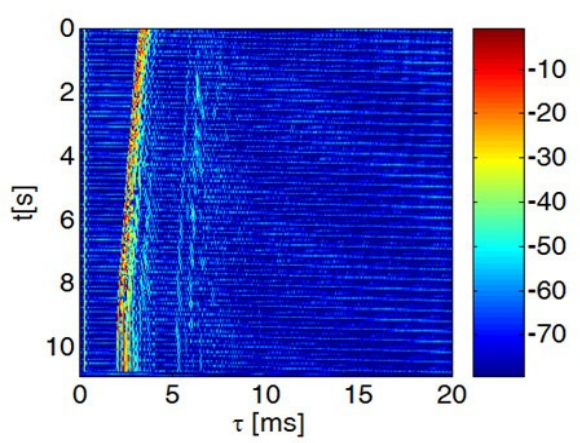

Fig 3. Time-varying Impulse response of UAC channel. 
Fig. 3 and Fig. 4 show the impulse response and the scattering function of the UAC channel measured over the course of an experiment in a lake. The receiving hydrophone was placed $0.5 \mathrm{~m}$ below the surface of the water and the speaker $-0.5 \mathrm{~m}$ deeper. The hydrophone was moved slowly with a velocity of $15 \mathrm{~cm} / \mathrm{s}$.

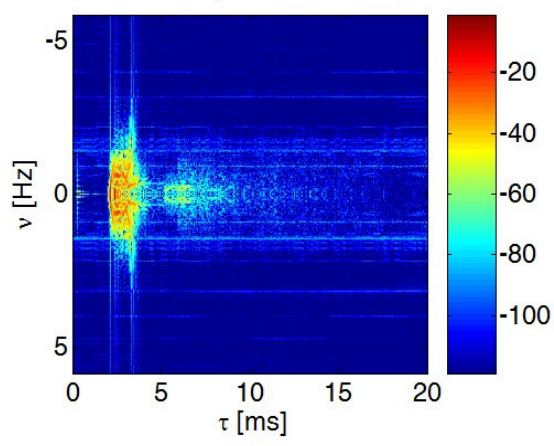

Fig 4. Scattering function of UAC channel.

As a result of integration $S(v, \tau)$ for the Doppler shift $v$ or delay $\tau$, one of two transmission characteristics is obtained. The first is the multipath intensity profile $P(\tau)$, describing the average received power variation as a function of time delay $\tau$ ( $\tau$ represents the signal's propagation delay that exceeds the delay of the first signal arrival at the receiver) [11]. For a shallow water channel, the received signal usually consists of numerous discrete multipath components (Fig. 5a). For deep water channels, due to strong refraction phenomenon, received signals are often seen as a continuum of multipath components.

For a single transmitted impulse, the time between the first and last received component represents the multipath delay spread $\tau_{M}$, during which the signal power falls to some threshold level below that of the strongest component. a)

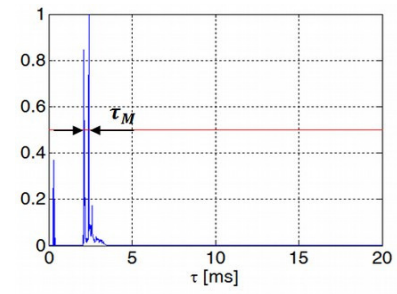

b)

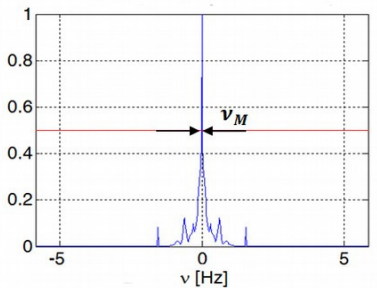

Fig 5. Multipath intensity profile (a) and Doppler power spectrum (b).

The second characteristic is the Doppler power spectral density $P(v)$, describing the time-variant nature of the channel. If the time-variation is random, it is seen as spectral broadening of $P(v)$, while a signal variation due to the transmitter or receiver movement with constant velocity reveals as a shift of $P(v)$ in $v$ domain. In case of UAC channel that fulfills the WSS assumption, $P(\boldsymbol{V})$ is a symmetrical function of $v$. However, measurement experiments have shown, that for non-WSS channels the Doppler power spectral density can have asymmetrical shape [18].
The width of $P(\boldsymbol{v})$ is referred to as maximum Doppler spread, denoted by $v_{M}$ (Fig. 5b).

On the basis of autocorrelation function $R_{h}(\Delta t, \Delta \tau)$, the autocorrelation of the channel transfer function is calculated as:

$$
R_{H}(\Delta t, \Delta f)=\int_{-\infty}^{\infty} R_{h}(\Delta t, \Delta \tau) e^{-j 2 \pi \Delta f \Delta \tau} d \Delta \tau
$$

where $-B / 2<\Delta f<B / 2$. It is a basis for calculating another two transmission characteristics of the communication channel. For $\Delta f=0$, the time correlation function $R_{h}(\Delta t)$ is obtained. Its width represents the coherence time $T_{C}$ (Fig. 6a). For $\Delta t=0$ the frequency correlation function $R_{h}(\Delta f)$ is obtained. It represents the correlation between the channel's response to two signals as a function of the frequency difference between the two signals. It allows to determine the coherence bandwidth $B_{C}$ as its width (Fig. 6b). a)

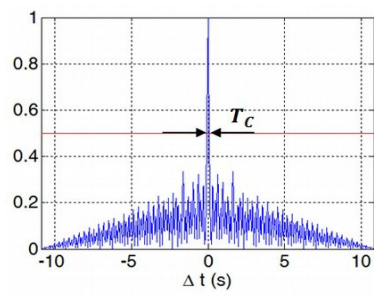

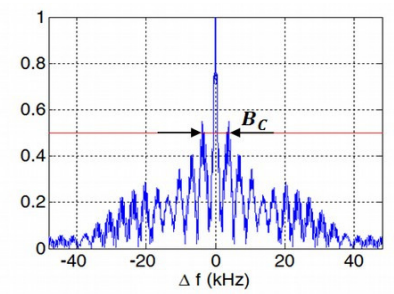

Fig 6. Time (a) and frequency (b) correlation functions.

The levels of transmission characteristics at which transmission parameters are determined depend on, amongst other factors, the technical capabilities of the particular UAC system and these are determined experimentally [11].

A set of four parameters $\left\{\tau_{M}, v_{M}, T_{C}, B_{C}\right\}$ is used for the designing a physical layer of the UAC system to minimize the influence of the time dispersion and time variability of the channel on the transmitted signal. The methodology is presented in the next section, in the examples of two UAC systems using different frequency diversity techniques.

\section{F. Limitations of WSSUS assumption}

In case of many underwater communication channels, especially when system terminals are in movement, the WSSUS model is of limited value. As it was shown in [18], UAC channels hardly ever fulfill the WSS and US assumptions, also in quasi-stationary conditions. But for a restricted period of time and a limited frequency range the assumptions can be satisfied. This approach is called localsense stationary uncorrelated scattering (LSSUS) [19]. It allows to use two other transmission parameters, namely stationary time $T_{D}$ and stationary bandwidth $B_{D}$, determining period of time and frequency range in which the WSSUS assumption is locally satisfied. They can extend the set of transmission parameters $\left\{\tau_{M}, v_{M}, T_{C}, B_{C}, T_{D}, B_{D},\right\}$ used for the designing a physical layer of the adaptive UAC system [19]. 


\section{Case Study - OFDM System}

The orthogonal frequency division multiplexing (OFDM) method is based on splitting the available transmission bandwidth into many narrow-band channels with center frequencies known as sub-carriers. The data is split into several parallel data streams, one for each sub-carrier, modulated with the use of a conventional modulation scheme (such as QAM, PSK or DPSK techniques) at a low symbol rate [11].

To adapt the OFDM signaling scheme to underwater channel conditions, the sub-carrier spacing $B_{\text {OFDM }}$ in the frequency domain is chosen, so as to satisfy the rule:

$$
B_{C}>B_{\text {OFDM }} \gg v_{M}
$$

The sub-carrier spacing should be smaller than the coherence bandwidth, wherein the transfer function remains constant. It should also be much greater than the maximum Doppler spread in order to avoid interference between neighboring sub-carriers due to the Doppler shift.

In the time domain, a single OFDM symbol duration $T_{\text {OFDM }}$ should satisfy the rule:

$$
\tau_{M}<T_{\text {OFDM }} \ll T_{C}
$$

The OFDM transmission symbol should be shorter than the coherence time $T_{C}$ in which the channel's statistics can be assumed to be stationary. On the other hand, the transmission symbol should last at least as long as the corresponding signal reflections take to reach the receiver. This prevents overlapping consecutive symbols, known as inter-symbol interferences. Moreover, each OFDM symbol is preceded by a cyclic prefix, i.e. a redundant repetition of the last segment of itself. The cyclic prefix of duration $T_{G}$ additionally protects the OFDM signal against inter-symbol interference [11].

Numerous sea and oceanic experimental trials of OFDM underwater communication systems have been reported [2027]. The data transmission rate of a few kbps is achieved in shallow water channels with a depth of several hundred meters. [23]. In case of a channel with a depth of less than $100 \mathrm{~m}$ (and thus with much stronger multipath propagation phenomenon), only few hundred kbps are obtained [20-21]. However, there is a lack of reported results for very shallow water channels with a depth of ca. $10 \mathrm{~m}$.

The OFDM technique is being implemented in a laboratory model of an acoustic data transmission system, designed at the Department of Marine Electronics Systems, Faculty of Electronics, Telecommunications and Informatics, Gdansk University of Technology [28-29]. Using this model, the underwater experiments were carried out in a lake. The underwater channel was ca. $4 \mathrm{~m}$ deep, and experiments were performed at distances from 1 to 30 $\mathrm{m}$. The speaker was placed 1 or $2 \mathrm{~m}$ below the surface and the hydrophone was placed $0.5,1$ or $2 \mathrm{~m}$ below the surface. In mobile scenarios, the hydrophone was moved slowly with a velocity of $15 \mathrm{~cm} / \mathrm{s}$. The analysis of the impulse response, measured using PRBS signal, has shown that the multipath delay spread, measured at a $-10 \mathrm{~dB}$ threshold level of the multipath intensity profile, was ca. $\tau_{M}=5 \mathrm{~ms}$ and the maximum Doppler spread was ca. $v_{M}=1 \mathrm{~Hz}$. The coherence bandwidth $B_{C}$ was measured as being the width of the frequency correlation function at level 0.7 of the maximum value, as well as the coherence time $T_{C}$, on the basis of the time correlation function. The results were: $B_{C}=23 \mathrm{~Hz}$ and $T_{C}=1 \mathrm{~s}$.

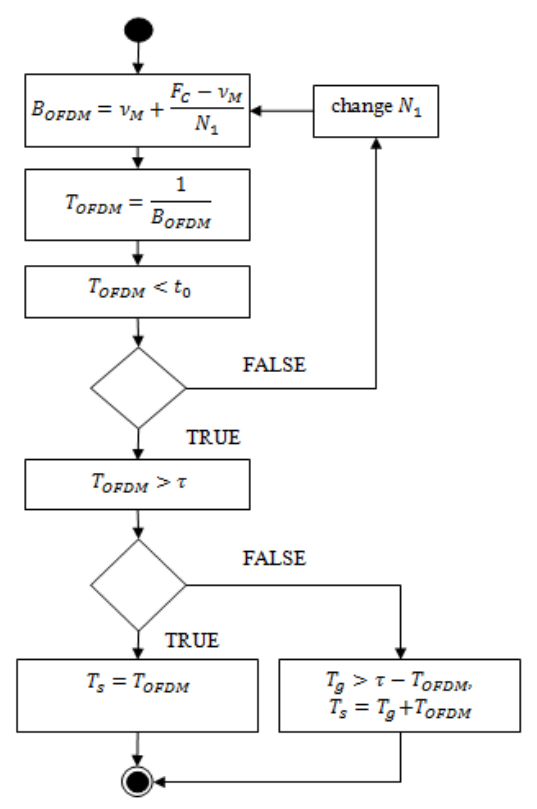

Fig 7. Procedure of calculating OFDM signaling scheme parameters.

The bandwidth of tested UAC system was $3 \mathrm{kHz}$, with a centre frequency of $5 \mathrm{kHz}$. With such fixed band and transmission parameters in the measured channel, the OFDM signaling scheme was determined as shown in Fig. 7. The sub-carrier spacing $B_{O F D M}$ was randomly chosen from a range of $\left(v_{M}, B_{C}\right)$. Next, the condition was checked, that is to say, if $T_{\text {OFDM }}<T_{C}, T_{\text {OFDM }}=1 / B_{\text {OFDM }}$. If this was not the case, another $B_{\text {OFDM }}$ value was randomly chosen. After adjusting the value of $B_{\text {OFDM }}$,the following condition was checked: $T_{\text {OFDM }}>\tau_{M}$. If this was not the case, a cyclic prefix of the duration $T_{G}=T_{\text {OFDM }} / 5$ was attached, lengthening the OFDM symbol duration.

With this procedure, a sub-carrier spacing of $B_{\text {OFDM }}=5.86 \mathrm{~Hz}$ was calculated that corresponds to symbol duration $T_{\text {OFDM }}=0.2 \mathrm{~s}$. Binary data, coded with a BPSK digital scheme, modulated each of the sub-carriers [18]. No channel coding was implemented. The maximum data transmission rate achieved during the underwater experiment in quasi-static scenario was 22 bps with bit error rate $B E R<10^{-3}$, and 880 bps with $B E R<10^{-1}$. In case of receiver moving in a uniform manner at a speed of about 15 $\mathrm{cm} / \mathrm{s}$, transmission rate of 615 bps was achieved with $B E R<10^{-1}$, however to perform any data transmission ( $<20$ bps) with $B E R<10^{-3}$, it was necessary to expand a sub-carrier spacing to $B_{O F D M}=11.72 \mathrm{~Hz}$. This confirms the strong influence of Doppler effect on UAC system performance. 


\section{Case Study - FHSS System}

The second considered communication system is based on the use of spread spectrum techniques [11]. These techniques create expedient conditions for the implementation of data transmission in occurrences of harsh multipaths. Where multipath delay spread of tens of milliseconds are encountered, this excludes the use of equalization. Spread spectrum techniques were originally developed for use in military systems on account of their low probabilities of interception (LPI) and decent resistance to different types of jamming signals.

The conception of spread spectrum systems is due to the well-known Shannon equation (13), to channel capacity C, with the specified bandwidth $\mathrm{B}$, and the power signal to noise ratio SNR:

$$
C=B \log _{2}(1+S N R)
$$

By transforming the above equations to the following form (14) we can concluded that the greater the noise that dominates the signal, the greater the possibility that it will require a wider bandwidth signal in order to receive it correctly.

$$
\frac{C}{B} \simeq 1.44 S N R
$$

Therefore, by greatly increasing the operating bandwidth, this at the same time, allows the use of a much lower signal to noise ratio.

In general, the work of the spread spectrum system is as follows. On the transmitter's side, this is carried out by transforming an information signal into a transmission signal with a much wider bandwidth. When this is the case, this is achieved by spreading the data signal with a pseudorandom code. On the receiver's side, there must be instated a despreading operation in order to restore the transmission signal to its original bandwidth. It is important to mention the same pseudorandom code in both the receiver and in the transmitter.

There are many types of spread spectrum techniques, of which the most common are: Frequency Hopping Spread Spectrum (FHSS), Direct Sequence Spread Spectrum (DSSS), Time Hopping Spread Spectrum (THSS) and Chirp Spread Spectrum (CSS). The first of them will be briefly discussed and analyzed.

Communication systems with frequency hopping spread spectrum techniques commonly use binary frequency shift keying. In this technique, all available channel bandwidth is divided into adjacent sub-channels. The carrier among subchannels is switched by using a pseudorandom sequence. Pseudorandom sequence controls the frequency synthesizer in every signaling interval. In this technique, MFSK modulation with non-coherent demodulation is usually employed. Because of this, the use of coherent modulations is difficult to retain phase coherence during the generating process of the signal, in accordance with the hopping pattern.

In transmitters, the data signal is subjected to channel encoding and interleaving. Then goes to the input of MFSK modulator. The modulator assigns a corresponding frequency in baseband to value of sending bit with duration
$T_{B}$. The produced signal is then placed in the appropriate sub-channel (frequency slot) for the time $T_{C}$ - termed as "dwell time". After the amplification, the signal passes on to the channel by using the transducer.

In the receiver, the amplified signal is firstly subjected to dehopping by mixing the synthesizer output with the received signal. Then the resulting signal is demodulated into an MFSK demodulator. A synchronization signal for keeping adequate synchronism in the pseudorandom generator with frequency hopping, a received signal is extracted from a received signal. A synchronization signal between each transmitted bit is placed in the signal frame. "Coarse" synchronization is carried out based on the signal of the frame synchronization which is usually a couple of LFM signals. This signal is also used in the estimation of channel impulse response functions. Hyperbolic frequency modulation (HFM) can be used because it is more resistant to the Doppler Effect.

For the sake of the simplicity in the receiving process, and by using the fast Fourier transform (FFT), non-coherent reception is performed. Similarly, the transform is the discrete-time implementation of the corresponding matched filters. In the next step, this is subjected to an analysis of the entire operating band, in which dehopping is carried out by selecting an appropriate sub-channel.

This technique has no impact on performance in an AWGN channel. The characteristic parameter for this type of spread spectrum system is gain processing, which expresses the bandwidth expansion factor:

$$
G_{p}=\frac{T_{B}}{T_{C}}
$$

In cases in which changes of the carrier frequency are used many times during particular data bit $\mathrm{T}_{\mathrm{B}}$, then we are dealing with a fast frequency hopping system (FFH). The figure below shows an example of an FFH system with the frequency hopping pattern $\mathrm{T}_{\mathrm{B}}=3 \mathrm{~T}_{\mathrm{C}}$.

Parameters such as coherence bandwidth and coherence time are included in the following manner. The FFH technique uses frequency and time diversity to effectively counteract the effects of the multipath. Due to the fact that this system commonly works for worst-case conditions in the channel, it is necessary to determine the requirements for the resistance to the maximum multipath delay spread. The cause of this, as shown in the figure above - i.e. channel clearing time - is the length of time long enough to allow the disappearance of multipath arrivals and in correspondence with the used pseudorandom time sequence during the transmission of a single bit. Although the extension of an applied pseudorandom sequence allows for more effective counteraction in the effects of the multipath, yet this will reduce the transmission rate and requires a broadening of the operating band. However, it should be noted that an important purpose of frequency diversity is the protection of each bit against spectral nulls due to frequency-selective fading.

The employed frequencies are spaced in order to take into account the limits of the frequency change caused due to the presence of the Doppler Effect in the entirety of the available operating band. They also satisfy the condition of or- 
thogonality by determining the minimum frequency separation $\Delta \mathrm{f}$ for the herein employed length of tones.

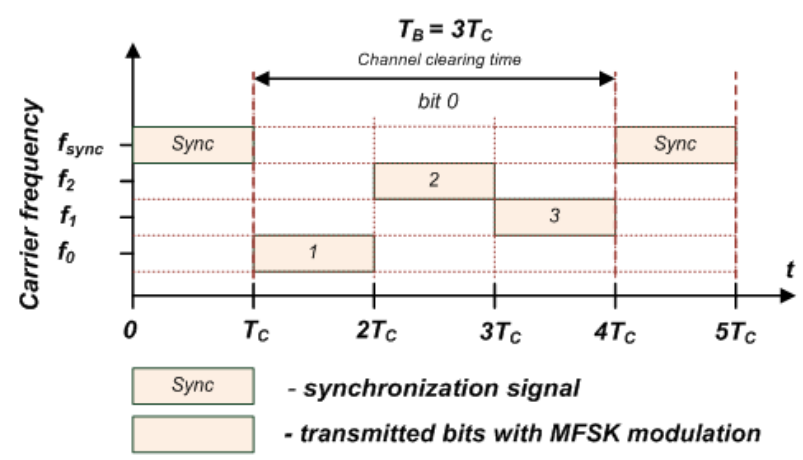

Fig 8. FFH system

The assumption concerning a communication system with this technique presented here can potentially reach maximum transmission of tens of bits per second. When the communication system takes into account the maximum value of a multipath delay spread of $30 \mathrm{~ms}$, then the system functioning according to the scheme shown in Fig. 8. has a value of a channel clearing time of $37.5 \mathrm{~ms}$, at a length $\mathrm{Tc}=$ $12.5 \mathrm{~ms}$. This yields a maximum transmission rate of 20bps for BFSK modulation, and 40bps for 4-FSK modulation, with no use of any channel coding.

Generally, a suppression mechanism in an interfering signal results from the fact that the useful signal is only transmitted at the specific moment in time, in a single narrowband channel. When the system works with the FFH technique, the interfering signal will coincide with the spectrum of the useful signal only to a small overall with its duration. The frequency hopping technique is simple to implement and is suitable in battery-powered solutions.

\section{Conclusions}

Due to the low propagation velocity of acoustic wave compared to electromagnetic wave, and thus greater influence of the Doppler effect, UAC systems provide transmission rates of tens or hundreds bps. The same modulation and coding techniques, applied in radiocommunication systems, allow to achieve transmission rate of Mbps and Gbps [30].

UAC channels are characterized by a large variety of propagation conditions. Designing reliable communication system requires knowledge of transmission parameters of the channel, namely multipath delay spread, Doppler spread, coherence time and coherence bandwidth. However, the possibilities of the measurement of transmission characteristics are limited, specially in case of overspread channels. New methods are needed to improve the estimation of time-varying dispersive underwater channels [12][18].

In shallow underwater channels with strong time-varying multipath propagation conditions the physical layer of data transmission is selected for the worst-case multipath delay spread and Doppler spread. The adaptation of the signaling scheme into the instantaneous transmission properties is possible only in selected well-tested channels. New modulation and coding techniques should be developed to perform reliable data transmission in time-varying propagation conditions [31].

\section{REFERENCES}

[1] J. Marszal, "Digital Signal Processing Methods Implemented in Polish Navy Sonar Modernization”, Polish Maritime Research, Vol. 21, 2014, No 2. pp. 65-75.

[2] J. Marszal, R. Salamon, "Distance Measurement Errors in Silent FMCW Sonar with Matched Filtering”, Metrology and Measurement Systems, Vol. XIX (2012), No. 2, pp. 321-332.

[3] R. Salamon, J. Marszal, W. Leśniak, "Broadband Sonar with a Cylindrical Antenna”, ACTA ACUSTICA united with ACUSTICA, Vol. 92, 2006, pp. 153-155.

[4] R. Salamon, J. Marszal, "Optimising the Sounding Pulse of the Rotational Directional Transmission Sonar”, ACTA ACUSTICA united with ACUSTICA, Vol. 88, 2002, pp. 666-669.

[5] J. Marszal, "Directivity pattern of active sonars with wideband signals", ACOUSTICAL IMAGING Vol. 19, 1992, pp. 915-919.

[6] J. Marszal, R. Salamon, A. Stepnowski, "Military sonar upgrading methods developed at Gdansk University of Technology”, Proc. IEEE Oceans'05 Europe Conference, Brest 2005, (Electronic document).

[7] I. Kochańska, "Considerations of adaptive digital communiations in underwater acoustic channel”, Hydroacoustics, Vol. 16, 2013, pp. 113120.

[8] J. Schmidt, "Reliable underwater communication system for shallow coastal waters”, Hydroacoustics, Vol. 17, 2014, pp. 171-178.

[9] J. Schmidt, "Underwater communication system for shallow water using modified MFSK modulation”, Hydroacoustics, Vol. 8, 2005, pp. 179184.

[10] R. Otnes, A. Asterjadhi, P. Casari, M. Goetz, T. Husøy, I. Nissen, K. Rimstad, P. van Walree, M. Zorzi, "Underwater Acoustic Networking Techniques”, SpringerBriefs in Electrical and Computer Engineering, 2012.

[11] B. Sklar, "Digital Communications: Fundamentals and Applications (2nd Edition)”, Prentice-Hall, 2001, pp. 944-996.

[12] I. Kochańska, "Adaptive identification of time-varying impulse response of underwater acoustic communication channel”, Hydroacoustics, Vol. 18, 2015, pp. 87-94.

[13] J., Marszal, R. Salamon, "Silent Sonar for Maritime Security Applications", Acoustical Society of America, Proceedings of Meetings on Acoustics, Vol. 17, 070082 (2013).

[14] J. Marszal, R. Salamon, L. Kilian, “Application of Maximum Length Sequence in Silent Sonar”, Hydroacoustics, Vol. 15, 2012, pp. 143-152.

[15] P. van Walree, "Channel sounding for acoustic communications: techniques and shallow-water examples”, Norwegian Defence Research Establishment (FFI), FFI-rapport 2011/00007

[16] P. A. Bello, "Characterization of randomly time-variant linear channels", IEEE Trans., Vol. CS-11, No 4, 1963, pp. 360-393.

[17] L. E. Franks, "Carrier and Bit Synchronization in Data Communiaction - A Tutorial Review”, IEEE Transactions on Communications, Vol. COM-28, No. 8, 1980 , pp. 1107 - 1121.

[18] I. Nissen, I. Kochańska, "Hydroakustik-Messung im Bornholmbecken zur lokalen Stationaritatsmodellierung beim Unterwasserschallkanal”, Fortschritte der Akustik - DAGA 2016, pp. 153-156.

[19] U. Chude-Okonkwo, R. Ngah, and T. Abd Rahman, "Time-scale domain characterization of non-WSSUS wideband channels," EURASIP Journal on Advances in Signal Processing, vol. 2011, no. 1, p. 123.

[20] F. Frassati, C. Lafon, L. P.A., and P. J.M., „Experimental assessment of OFDM and DSSS modulations for use in littoral waters underwater acoustic communications," in Oceans'05 Proc. MTS/IEEE, France, 2005.

[21] S. Coatelan and A. Glavieux, „Design and test of coding OFDM system on the shallow water acoustic channel," in Oceans '95 Proc.. MTS/IEEE, Brest, France, 1995.

[22] R. Bradbeer, E. Law, and E. Yeung, „Using multi-frequency modulation in a modem for the transmission of near realtime video in an underwater environment,” in Consumer Electronics, 2003. ICCE., Hong Kong

[23] M. Chitre, S. H. Ong, and J. Potter, „Performance of coded OFDM in vary Shallow water channels and snapping shrimp noise,” in Ocenas '05 Proc. MTS/IEEE , Singapore, 2005.

[24] M. Stojanovic, „Low Complexity OFDM Detector for Underwater Acoustic Channels," in IEEE Oceans'06 Proc. MTS/IEEE, Boston, MA, 2006. 
[25] B. Li, S. Zhou, M. Stojanovic, L. Freitag, and P. Wille, „Multicarrie Communication over Underwater Acoustic Channel with non uniform Doppler shifts," IEEE Journal of Oceanic Engineering, vol 33, nr 2, pp. 198-209, 2008.

[26] B. Li, S. Zhou, M. Stojanovic, and Freitag, „Pilot-tone based ZP OFDM demodulation for an Underwater acoustic channel," in Oceans'06 MTS/IEEE, Boston, MA, 2006.

[27] T. Suzuki, H. M. Tran, and T. Wada, "An underwater acoustic OFDM communication system with shrimp (impulsive) noise cancelling," in Proc. of the International Conference on Computing, Management and Telecommunications (ComManTel '14), pp. 152-156, IEEE, D Nang, Vietnam, April 2014.
[28] I. Kochańska, H. Lasota, "Investigation of underwater channel timevariabiliy influence on the throughput of OFDM data transmission system", Proceedings of Meetings on Acoustics, POMA, 17, Acoustical Society of America, 2013.

[29] I. Kochańska, "Measurements of transmission properties of acoustic communication channels", Hydroacoustics, Vol. 15, 2012, pp. 91-98.

[30] A. Matoba, M. Hanada, M.W. Kim, "Throughput Improvement by Adjusting RTS Transmission Range for W-LAN Ad Hoc Network", Proceedings of the 2014 Federated Conference on Computer Science and Information Systems, 2014, pp. 941-946.

[31] I. Nissen, "Measurements of transmission properties of acoustic communication channels", Hydroacoustics, Vol. 18, 2015, pp. 113-126. 